\title{
A Differential Thermal Analysis Apparatus for Exploring the Glass Transition
}

\author{
William R. Heffner \\ International Materials Institute for Glass, Lehigh University, 7 Asa Drive, Bethlehem, PA 18015
}

\begin{abstract}
Glass is among the most common materials in our everyday lives. And yet the science behind this interesting, complex and ubiquitous material is seldom considered in the undergraduate science curriculum. The glass transition ( $\mathrm{T}_{\mathrm{g}}$ ) is both a fundamental and defining concept in understanding the glassy state. To facilitate the experimental exploration of this important topic we have developed a simple home-built apparatus for measuring the $\mathrm{T}_{\mathrm{g}}$ and the associated relaxation phenomena. The simple differential thermal analysis (DTA) apparatus requires only basic mechanical and electronic construction skills yet provides excellent resolution of the $\mathrm{T}_{\mathrm{g}}$ for the low temperature sugar glasses examined. We also demonstrate the strong effect of thermal history on $T_{g}$, including both the effect of cooling rate and aging. This apparatus provides an interesting and intuitive path to the student's exploration and understanding of the glassy state and provides a resource for deeper, independent and open-ended study of the relaxation phenomena, especially appropriate for an advanced undergraduate laboratory.
\end{abstract}

Keywords: glass transition, DTA, calorimeter, relaxation, education, physics, material science.

PACS: 001.50.Pa, 7.20, 07.50.-e

\section{INTRODUCTION}

A quick glance around the home or workplace will provide numerous examples of the glass technology pervading our lives from cookware to low-e glass windows; from the complex lenses on our face, to the fracture resistant displays on our devices; not to mention the glass fibers insulating our homes and enabling the high speed internet on which we are so dependent. All of these applications and many others depend on the unique properties of glass, which in turn depend on the structure, or lack thereof, within this non-crystalline, liquid-like, yet solid state. Glass results from cooling a liquid "fast enough" to avoid crystallization. On cooling the viscosity of these supercooled liquids increase dramatically. At some point the viscosity within the cooling liquid becomes so great that the changes and contraction within its structure can no longer keep up with the rate of temperature decrease and the structure becomes "frozen" in time. This point is referred to as the glass transition, although it is not an actual phase transition and there is not a single well-defined temperature [1]. Thermal response parameters such as heat capacity and thermal expansion will exhibit significant increases above the frozen, glassy region and the abrupt changes provide a practical mark or definition of the glass transition temperature $\left(\mathrm{T}_{\mathrm{g}}\right)$.

While glass usually receives little or no attention in the general physics or chemistry curriculum, it has, nonetheless, been an area of active research in both materials and condensed matter physics communities for decades. In fact, in 1995 the Nobel Prize laureate
Philip Anderson opined that "The deepest and most interesting unsolved problem in solid state theory is probably the nature of glass and the glass transition."[2]. An excellent review describing the glass transition phenomena and unsolved issues was prepare by Dyre[1] in 2006 and the sentiment was even reiterated in a popular New Your Times article by Chang [3] in 2008, entitled "The Nature of Glass Remains Anything but Clear". From both a technological and a theoretical perspective, the fundamental principles of glass science remain too relevant and interesting to be left out of our undergraduate science education.

To this end, we have been developing a series of experiments for the quantitative exploration of glass science fundamentals, using readily accessible materials and home-built apparatus [4, 5]. For many of these experiments we examine glasses made from sugars and the artificial sweetener Isomalt (a sugar alcohol), which the student can prepare on a kitchen stove. In this paper we turn our attention to a consideration of the glass transition phenomenon and relaxation. Thermal analysis is one of the important tools for studying changes in materials such as melting, crystallization and the glass transition temperature $\left(\mathrm{T}_{\mathrm{g}}\right)$ and its associated relaxation phenomena [6]. These measurements generally rely on differential techniques to enhance their sensitivity, monitoring the difference in response (e.g. length, temperature, heat flow, etc.) between a reference and the sample while both are heated (or cooled) in the same enclosure. DSC (differential scanning calorimetry) measures heat flow directly and is the most common technique for measuring $\mathrm{T}_{\mathrm{g}}$. However, such instruments 
are expensive and generally unavailable outside of the specialized materials research laboratory. Differential thermal analysis (DTA), however, is simpler to implement and provides essentially the same information. The apparatus requires only basic mechanical and electronic skills to construct, and provides a good opportunity for the student to participate in both the experimental design and construction. In the sections to follow we describe the DTA apparatus and report measurements of the glass transition for the Isomalt glass system, including the effect of different thermal histories, from slow cool to quench, as well as the effect of aging.

\section{EXPERIMENTAL APPARATUS}

Our DTA consists of monitoring the temperature difference between the sample and a reference material, placed in separate test tubes, while they are simultaneously heated in a surrounding enclosure. An aluminum block with an embedded cartridge heater(s) provides the temperature ramped enclosure. Both the differential and block temperatures are monitored as the block is heated. A sketch of the apparatus is shown to the left in Fig. 1. The differential temperature provides

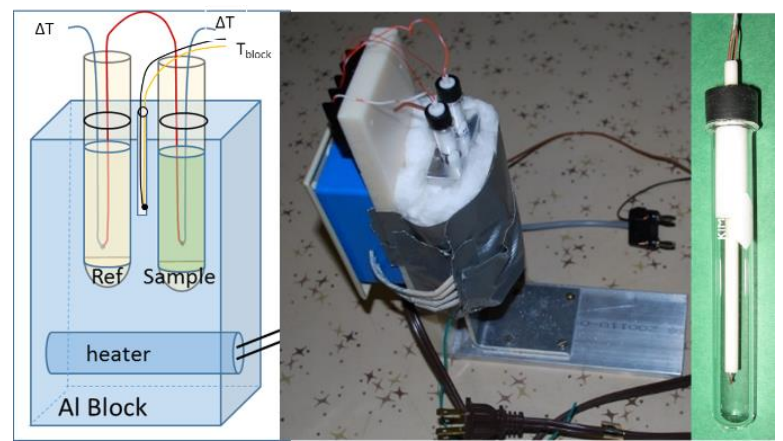

Figure 1. Sketch of the home-made DTA apparatus (left). Photo of the actual apparatus in the middle and the fixed position thermocouple probe is shown on the right.

a sensitive monitor of thermal events occurring within the sample during the heating (or cooling) process. Endothermic (heat absorbing) events such as melting will result in the sample temperature $\left(\mathrm{T}_{\mathrm{s}}\right)$ falling below the reference temperature $\left(\mathrm{T}_{\text {ref }}\right)$ resulting in a negative deviation of the differential temperature $\left(\Delta T=T_{s}-T_{\text {ref }}\right)$, persisting until the event is complete. Thus for melting a negative going peak will be observed on heating. The glass transition is associated with an abrupt increase in the apparent heat capacity around $\mathrm{T}_{\mathrm{g}}$ and thus a step change in $\Delta \mathrm{T}$ is expected.

In an earlier implementation [5] we utilized a heated oil bath instead of the aluminum block. While the aluminum block requires a little more machining, it eliminates the use of hot oil, averting safety and fire concerns, and it facilitates both temperature control and cooling options. Cooling is achieved through an aluminum mounting bracket, attached to the heating block through partially insulating Teflon spacers. The base of the holder can be placed in an ice bath to achieve sub-ambient temperatures and to adjust the cooling rate.

The aluminum block for our apparatus was made from standard aluminum bar stock (multipurpose 6061 alloy, 1.90 x $5.08 \mathrm{~cm}$ (3/4”x 2")) designed to accommodate two $10 \times 75 \mathrm{~mm}$ standard Pyrex test tubes, with holes drilled to depth of $5 \mathrm{~cm}$ (2") and spaced 1.9 cm $(3 / 4$ ") apart, with two horizontal 50 W cartridge heaters embedded below the test tubes. The heaters were powered through a variable voltage transformer for simple manual adjustment of the heating rate.

Thermocouples (TC) are used to measure both the differential and block temperatures. The differential TC pair is made from a single piece of constantan wire soldered at each end to thin copper hookup wires. Calibration data for this type $\mathrm{T}$ thermocouple can be obtained from standard tables or a value of $0.042 \mathrm{mV} /$ ${ }^{\circ} \mathrm{C}$ can be used. In order to keep the TC probe placement fixed during measurements we thread the TC wires through a piece of dual bore ceramic tubing held in alignment by a cylindrical Teflon rod with cap as shown to the right in the Fig. 1. This additional feature is not essential but does enhance the repeatability of the measurements.

A low cost instrumentation amplifier IC (INA 122) provides the high gain needed to bring the small (microvolt) differential TC signals into a range $( \pm 5 \mathrm{~V})$ reasonable for measurement with an ordinary DVM or data logger. The output is fed to a second filter stage to remove unwanted stray AC pickup noise. The circuit is shown in Fig. 2. With an overall gain of 8000,

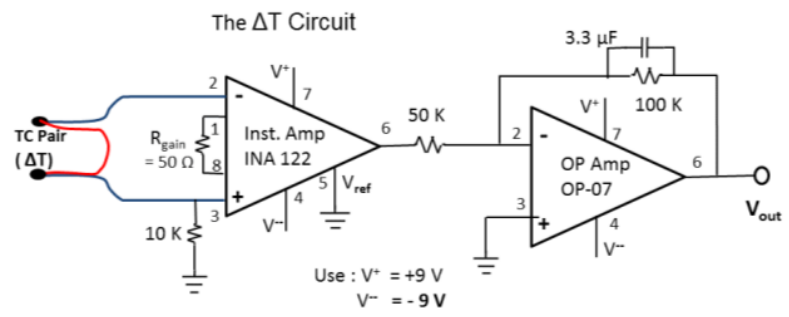

Figure 2. Two stage circuit for amplification and filtering of the signal from the differential thermocouple pair.

the circuit output is approximately $0.335 \mathrm{~V} /{ }^{\circ} \mathrm{C}$. The block temperature is monitored using a standard $\mathrm{K}$ type thermocouple connected to an AD595 IC. The AD595 provides an internal, temperature compensated, ice point reference for the thermocouple and outputs a voltage calibrated directly with the temperature (at 10 $\mathrm{mV}$ per ${ }^{\circ} \mathrm{C}$ ). 
Automated data acquisition is very useful, almost essential, for high quality capture of the various thermal events. For experimenters who do not already have their own data logging platforms, a simple, low-cost alternative is the DI-149 USB Data Acquisition unit (from Dataq Instruments). This device in conjunction with their Windaq-XL software was used to bring the data directly into an Excel spreadsheet, where the data was displayed graphically as it was taken. Active monitoring of the experiment is quite useful and allows one to adjust the heating rate as needed and adapt quickly to any issues that may arise.

The DTA is suitable to examine melting, crystallization or glass transitions over a range from slightly above room temperature to about $160{ }^{\circ} \mathrm{C}$. Higher temperature can degrade organic sample and reference materials. We use vegetable oil as our reference material. Steric acid provides an excellent crystallization standard, with a melting point near $70^{\circ} \mathrm{C}$. Sucrose based sugar glasses[4] and the artificial sweetener, Isomalt, provide two low $\mathrm{T}_{\mathrm{g}}$ glasses that can be made in the kitchen. The $T_{g}$ of these sugar-based glasses will vary with water content, which in turn is determined by the final boiling temperature. Isomalt glass is less hydroscopic than the sucrose based glasses and can be prepared with the higher $\mathrm{T}_{\mathrm{g}}$, and thus is the sample examined in this paper. A commercial grade ClearCut ${ }^{\mathrm{TM}}$ Isomalt (from makeyourownmolds.com ) was used. The granular Isomalt was mixed with 15 wt. $\%$ water and heated until dissolved. Continued boiling until $170{ }^{\circ} \mathrm{C}$ removes sufficient water to achieve a room temperature glass with glass transition near $40^{\circ} \mathrm{C}$. The hot Isomalt solution is poured into the Pyrex test tubes, filling to about the half level. The thermocouple probe must be inserted while the sample is hot and fluid. If the probe is inserted later, a heat gun or hot oil bath can be used to reheat the sample in the test tube.

For this study we prepare the same sample under different cooling conditions, from quenched to slow cooling, and with different amounts of aging. Heating runs were made at about $3{ }^{\circ} \mathrm{C} / \mathrm{min}$ to a maximum of 140 ${ }^{\circ} \mathrm{C}$, follow by a more gradual cool down to room temperature once the heater power is turned off. Quenching was done by removing the test tube of Isomalt at $140^{\circ} \mathrm{C}$ and immersing quickly into an ice bath for at least an hour.

\section{RESULTS}

Both the differential response $(\Delta \mathrm{T})$ and bath temperature are shown vs. time for the quenched Isomalt sample in Fig. 3. For the differential response we report the amplified differential thermocouple voltage which serves as a relative $\Delta \mathrm{T}$ measurement and we will use the terms interchangeably in what follows. Ice cooling of the base was used to achieve a sub-ambient start temperature. That the sample was well equilibrated was established by observing a flat baseline behavior in both the $\mathrm{T}$ and $\Delta \mathrm{T}$ signals prior to heating. In the figure, heating began after a few minutes, after which it takes about 2.5 minutes to achieve a constant slope, indicative of steady state response. Note the nearly constant heating rate of about $2.8{ }^{\circ} \mathrm{C} / \mathrm{min}$ that was achieved through simple manual adjustment of the heater voltage. While this time plot is useful to ascertain the conditions, the proper DTA trace consists of plotting the differential response vs. the temperature as shown in the lower portion of Fig. 3. Notice the clear step-like change that occurs near $40{ }^{\circ} \mathrm{C}$ against a generally upward sloping and constant background. This step is characteristic of a the glass transition of a non-aged sample, although generally the baselines are adjusted to be flat.

We find that the most repeatably linear portions of the curve to occur right above the glass transition where the sample is quite stiff and this facilitates a simple baseline adjustment (flattening) within the Excel spreadsheet. Deviation from linearity above about $70^{\circ} \mathrm{C}$ are occasional and attributed to movement of the thermpocouple position that can occur once the material softens.

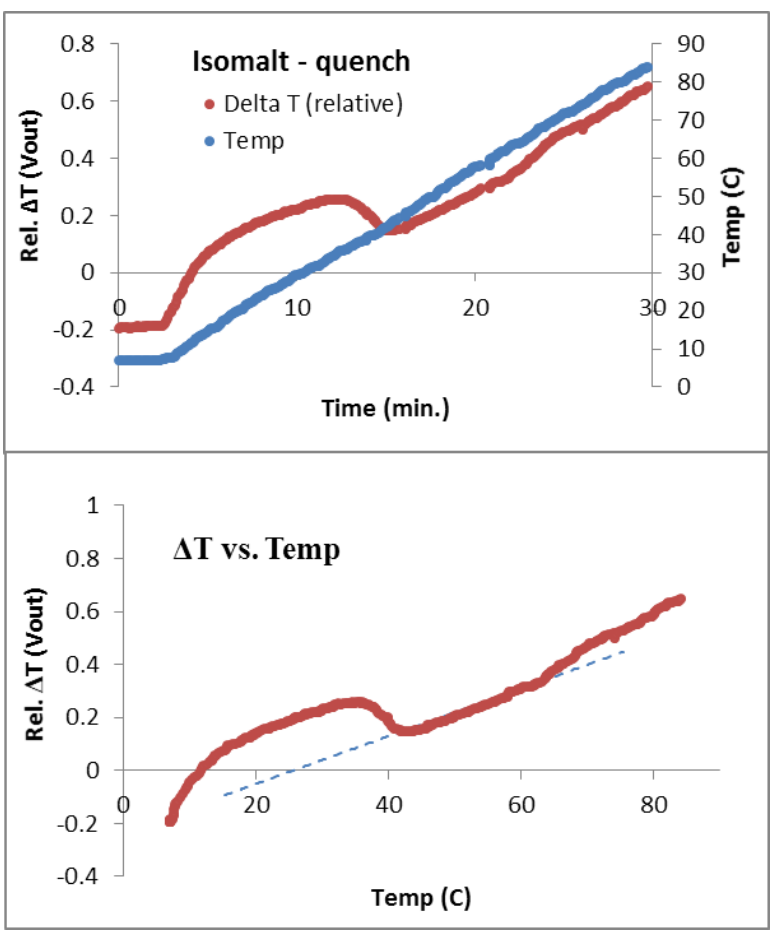

Figure 3. (Above) Plot of the relative $\Delta \mathrm{T}$ (as output voltage from amplifier) and block temperatures vs. time for quenched Isomalt. (Below) The more conventional $\Delta \mathrm{T}$ vs. $\mathrm{T}$ plot for the same data. Dashed line indicates baseline slope to be corrected.

In Fig. 4 we compare the baseline flattened DTA response of the quenched sample with samples cooled 
more slowly and allowed to rest (aged) for various amounts of time from one hour to three days. For the quenched sample the simple step is observed at $\mathrm{T}_{\mathrm{g}}$. The $\mathrm{T}_{\mathrm{g}}$ value is typically taken as the midpoint value, which is about $40^{\circ} \mathrm{C}$ in our example.

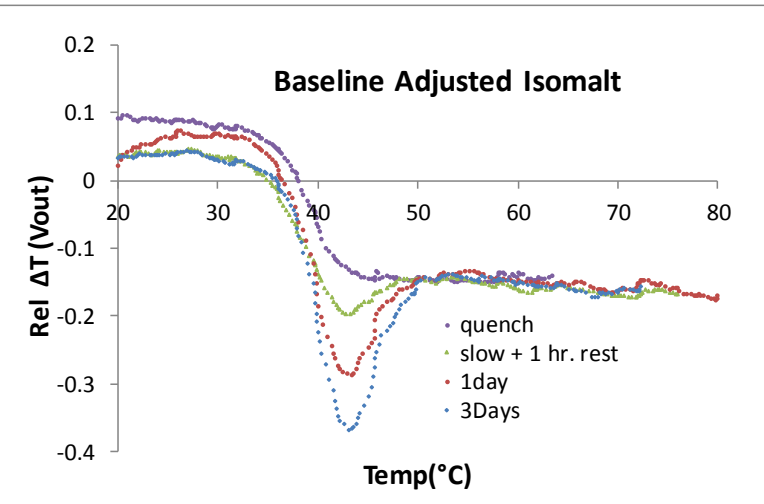

Figure 4. Baseline flattened data for the same sample of Isomalt under four different thermal histories.

In contrast, the non-quenched samples all show the emergence of a post- $\mathrm{T}_{\mathrm{g}}$ endothermic peak, associated with the varying amounts of relaxation which took place in the glassy structure both during and after cooling. The occurrence and association of these endothermic peaks was originally observed by Tool [7] in 1946. The detailed quantitative understanding of the dynamics took place over the next three decades.

In short, the peak arises because the aged glass has relaxed to a lower enthalpy state with an associated increase in its inherent relaxation time. On reheating the more relaxed structure of the aged material responds more sluggishly to the ramped temperature change than an unaged sample. When the sample's relaxation rate is slow relative to the heating rate, its enthalpy change can lag and fall below the metastable equilibrium state of the supercooled liquid. On further heating, this enthalpy undershoot eventually reverts back to the equilibrium condition accompanied by an endothermic event. The size of the peak reflects the amount of relaxation that has taken place. This interesting topic certain warrants a much longer discussion than can be provided here. A good review of both the history and the technical understanding of this topic can be found in a text by Scherer [8]. The phenomena and analysis remain widely used tools today in the study of relaxation and aging in both glass and polymers.

\section{SUMMARY}

In this paper we have highlighted the importance of glass to our modern technologies and the significant questions it raises concerning our understanding of matter. Central to understanding glass are the glass transition and its underlying relaxation phenomena. To facilitate an empirical approach to this understanding we have designed and described a low-cost, home-built DTA capable of measuring the glass transition phenomena in simple low temperature glasses made from sugar and artificial sweeteners. We demonstrate how the apparatus can be used to engage in serious, quantitative experiments to examine the effect of thermal history and aging in glassy materials using this very accessible system. This apparatus provides a hands-on, intuitive path to engage the student in glass science and experimental design. In addition the apparatus could also provide a resource for deeper, open-ended and independent study of relaxation phenomena, especially appropriate for an advanced undergraduate laboratory.

\section{ACKNOWLEDGMENTS}

This work was supported by the National Science Foundation through the International Materials Institute for New Functionality in Glass, IMI Grant DMR 0844014 .

\section{REFERENCES}

1. J. C. Dyre, Rev. Mod. Phys. 78, 953 (2006).

2. P.W. Anderson, Science 267, 1615 (1995).

3. K. Chang (July 29, 2008). "The Nature of Glass

Remains Anything but Clear", New York Times.

Retrieved from http://www.nytimes.com

4. W. R. Heffner and H. Jain, "Building a Low Cost, Hands-on Learning Curriculum on Glass Science and Engineering using Candy Glass" in MRS Proceedings 1233 , Boston, 2009, edited by M. M. Patterson (Materials Research Society, 2010).

5. W. R. Heffner and H Jain, "Low-Cost, Experimental Curriculum in Materials Science Using Candy Glass

Part 2: Home-Built Apparatuses" in MRS Proceedings

1657, Boston, 2014, (Materials Research Society, 2014).

6. J.E. Shelby, An Introduction to Glass Science and Technology, $2^{\text {nd }} E d$. (The Royal Society of Chemistry, Cambridge, 2005) p237.

7. A. Q. Tool, J. Am. Ceram. Soc. 29, 240 (1946).

8. G. W. Scherer, Relaxation in Glass and Composites (Krieger Publishing Co, Malabar, 1992). 